\title{
1 Characterisation of Medicago truncatula CLE34 and CLE35 in
}

\section{2 nodulation control}

3

4

5

6

7

8

Authors: Celine Mens ${ }^{1}$, April H. Hastwell ${ }^{1}$, Huanan Su ${ }^{1,2}$, Peter M. Gresshoff ${ }^{1}$, Ulrike Mathesius ${ }^{3}$, Brett J. Ferguson ${ }^{1 *}$

Affiliation: ${ }^{1}$ Integrative Legume Research Group, School of Agriculture and Food Sciences, The University of Queensland, St Lucia, Brisbane 4072, Queensland, Australia.

${ }^{2}$ National Navel Orange Engineering Research Center, School of Life Science, Gannan Normal University, Ganzhou 341000, China.

${ }^{3}$ Division of Plant Sciences, Research School of Biology, Australian National University, Canberra 2601, ACT, Australia

*Corresponding Authors: Brett J. Ferguson, address as above, Phone: +61-7-3346 9951, email: b.ferguson1@uq.edu.au

Running head: Rhizobia- and nitrate-induced CLE peptides of $M$. truncatula

Word count: 3718

Number of figures: 4 
Abstract

Legume plants form a symbiosis with $\mathrm{N}_{2}$-fixing soil rhizobia, resulting in new root organs called nodules that enable $\mathrm{N}_{2}$-fixation. Nodulation is a costly process that is tightly regulated by the host through Autoregulation of Nodulation (AON) and nitrate-dependent regulation of nodulation. Both pathways require legume-specific CLAVATA/ESR-related (CLE) peptides. Nitrogen-induced nodulationsuppressing CLE peptides have not previously been characterised in Medicago truncatula, with only rhizobia-induced MtCLE12 and MtCLE13 identified. Here, we report on novel peptides MtCLE34 and MTCLE35 in nodulation control pathways. The nodulation-suppressing CLE peptides of five legume species were classified into three clades based on sequence homology and phylogeny. This approached identified MtCLE34 and MtCLE35 and four new CLE peptide orthologues of Pisum sativum. Whereas MtCLE12 and MtCLE13 are induced by rhizobia, MtCLE34 and MtCLE35 respond to both rhizobia and nitrate. MtCLE34 was identified as a pseudogene lacking a functional CLE-domain. Overexpression of MtCLE12, MtCLE13 and MtCLE35 inhibits nodulation. Together, our findings indicate that MtCLE12 and MtCLE13 have a distinct role in AON, while MtCLE35 regulates nodule numbers in a rhizobia- and nitrate-dependent manner. MtCLE34 likely had a similar role to MtCLE35 but its function was lost due to a nonsense mutation resulting in the loss of the mature peptide.

Keywords: autoregulation of nodulation, CLE peptide, legumes, nitrate, nodulation, plant signalling and development, symbiosis 


\section{Introduction}

Nitrogen availability is essential for plant growth, but is highly variable in the rhizosphere with up to 1,000-fold differences in nitrate concentration ( $\mu \mathrm{M}-\mathrm{mM})$ reported within a four-meter diameter (Miller et al., 2007). Plants encountering nitrogen deficiency require an increase in root plasticity to maintain an optimal nitrogen balance (i.e., via lateral root growth, increased uptake or soil microbial interactions). Legume plants have established a symbiotic relationship with rhizobia bacteria that fix atmospheric nitrogen $\left(\mathrm{N}_{2}\right)$ for plant-use in return for photosynthetic carbohydrates. This requires the formation of specialised root nodules that house the bacteria. Biological nitrogen fixation and nodule organogenesis are both resource intensive processes and thus the host plant has evolved regulatory mechanisms to control nodule numbers, such as the Autoregulation of Nodulation (AON) pathway (Caetano-Anollés \& Gresshoff, 1991a, 1991b; Delves et al., 1986; Ferguson et al., 2019).

AON is a systemic feedback mechanism requiring extensive signalling between the roots and shoot Moreover, when ample nitrogen is present in the soil, nodulation is negatively controlled via nitratedependent regulation of nodulation which has many signals in common with AON. In fact, the first AON mutants were identified as nitrate-tolerant supernodulators (nts) (Carroll et al., 1985). Both processes initially require upregulation of CLAVATA3/EMBRYO SURROUNDING REGION-RELATED (CLE) peptides that are perceived by a leucine-rich repeat receptor-like kinase (LRR-RLK) called MtSUNN/GmNARK/LjHAR1/PsSYM29/PvNARK (Krusell et al., 2002; Nishimura et al., 2002; Searle et al., 2003; Schnabel et al., 2005; Ferguson et al., 2014). The shoot-derived signal of AON has been identified as miR2111, which targets the Kelch-repeat factor, TOO MUCH LOVE (TML), for degradation to inhibit the development of new nodules (Takahara et al., 2013; Tsikou et al., 2018; Gautrat et al., 2020). Recent findings using Lotus japonicus suggest TML might not be required for nitrate-dependent inhibition (Nishida et al., 2020).

CLE peptides are hormone-like regulators of cell division and differentiation acting in local and systemic cell-to-cell communication. Mature CLE peptides are 12 to 13 amino acids in length and are post-transcriptionally processed from the C-terminal region of their respective pre-propeptides (Hastwell et al., 2015b). They require further modification through triarabinosylation of a central hydroxylated proline residue by members of the hydroxyproline $\mathrm{O}$-arabinosyltransferase family (Okamoto et al., 2013; Corcilius et al., 2017; Kassaw et al., 2017; Hastwell et al., 2019).

The first CLE peptide was identified for its role in maintaining the stem cell population of the shoot apical meristem; a process during which the peptide AtCLV3 binds to the LRR-RLK AtCLV1 receptor 
(Clark et al., 1995; Fletcher et al., 1999). Since then, CLE peptides have been identified for having roles in the root apical meristem (CLE40), vasculature differentiation (TDIF peptides) and inhibition of lateral root development (Hobe et al., 2003; Ito et al., 2006; Araya et al., 2014). A number of CLE peptides are also reported to respond to environmental factors including rhizobia, mycorrhiza, nitrate and phosphate, making them essential for regulating critical plant-microbe interactions as well as macronutrient homeostasis and acquisition (Okamoto et al., 2009; Mortier et al., 2010; FunayamaNoguchi et al., 2011; Reid et al., 2011; Araya et al., 2014; de Bang et al., 2017; Karlo et al., 2020).

Nodulation-suppressing CLE peptides are expressed when soil nitrogen availability is high and/or following rhizobia inoculation. They are unique to legumes and have been characterised across different model plants including Medicago truncatula, L. japonicus, common bean (Phaseolus vulgaris) and soybean (Glycine max) (Okamoto et al., 2009; Mortier et al., 2010; Reid et al., 2011; Lim et al., 2011; Ferguson et al., 2014; Lim et al., 2014; Nishida et al., 2016). The rhizobia-responsive CLE peptides (MtCLE12 and MtCLE13, GmRIC1 and GmRIC2, PVRIC1 and PVRIC2, and LjCLE-RS1, LjCLE-RS2, LjCLERS3) are induced in the root following inoculation, and their overexpression results in the inhibition of nodule numbers.

To date, nitrate-responsive nodulation-suppressing CLE peptides have not been identified in $M$. truncatula as they have for soybean (GmNIC1a and GmNIC1b), common bean (PvNIC1) and L. japonicus (LjCLE-RS2, LjCLE-RS3 and LjCLE40). Bioinformatic analyses by Hastwell et al. (2017) identified a total of 52 CLE peptide encoding genes within the $M$. truncatula genome, with the uncharacterised MtCLE34 and MtCLE35 grouping with the previously identified MtCLE12 and MtCLE13 and similar rhizobia- and nitrate-responsive CLE peptides in other model legumes (Hastwell et al., 2015b; 2017). Our findings reported here have established new roles for the M. truncatula CLE peptides MtCLE34 and MtCLE35 in nitrogen- and rhizobia-signalling during nodulation control.

\section{Material and Methods}

Plant and bacterial growth conditions

Seeds of wild-type Medicago truncatula Jemalong A17 were scarified in $98 \%$ sulphuric acid and surface sterilised using $6 \%(\mathrm{w} / \mathrm{v})$ sodium hypochlorite followed by extensive rinsing in sterile $\mathrm{H}_{2} \mathrm{O}$. Seedlings were subsequently grown on agar plates containing nitrogen-free Fåhraeus medium (Fåhraeus, 1957) in controlled growth chamber conditions with a photoperiod of $16 \mathrm{~h} / 8 \mathrm{~h}$ at $23^{\circ} \mathrm{C}$. Inoculation with 
Sinorhizobium meliloti $1021\left(\mathrm{OD}_{600}=0.05\right)$ was staggered so that all different time points could be harvested at the same time. Solid Bergersen's Modified Medium (BMM) was used to culture rhizobia at $28^{\circ} \mathrm{C}$ for 2 days and single colonies were grown overnight in liquid BMM before seedling inoculation (Gresshoff et al., 1977). The nodulation-susceptible root zone was harvested at 0, 1, 2, 3, 5 and 7 days post inoculation (dpi).

For nitrate treatments, wild-type plants were grown in a sand:perlite (1:2) mixture in controlled growth chamber conditions with a $16 \mathrm{~h} / 8 \mathrm{~h}$ photoperiod at $24^{\circ} \mathrm{C}$ and $21^{\circ} \mathrm{C}$ respectively. Plants were watered as required with nitrogen-limiting Fåhraeus medium (Fåhraeus, 1957) for the first week and supplemented with $0 \mathrm{mM}, 2 \mathrm{mM}$ or $10 \mathrm{mM}$ of nitrate $\left(\mathrm{KNO}_{3}\right)$ every second day in the week before sampling the total root.

Gene expression analysis

Entire roots of 14 day-old nitrate-treated plants or the nodulation-susceptible zone of 11-day old inoculated plants were harvested, snap-frozen and homogenised in liquid nitrogen. The total RNA was extracted using the Maxwell ${ }^{\circledR}$ LEV Simply RNA Tissue kit (Promega) or the RNeasy Plant Mini Kit (Qiagen) according to the manufacturer's protocol. The quality and quantity of each RNA sample were assessed using the NanoDrop One Spectrophotometer ${ }^{\mathrm{TM}}$ (Thermo Fischer Scientific). cDNA was generated using SuperScript ${ }^{\circledR}$ III Reverse Transcriptase (Invitrogen) from 500 ng of DNase-treated RNA. RT-qPCR analysis was conducted using the Roche LightCycler ${ }^{\circledR} 96$ or the Bio-Rad CFX384 Touch ${ }^{\text {TM }}$ with GoTaq SYBR green fluorescence detection (Promega). All reactions were performed in duplicate for at least two biological replicates $(n=11-14)$ and a target amplicon size of approximately $100 \mathrm{bp}$. The constitutively expressed Mt40S ribosomal S8 protein was included to normalise expression levels (Mortier et al., 2010).

\section{Bioinformatic analysis}

To genetically characterise MtCLE34 and MtCLE35 further, a multiple sequence alignment was performed using Clustal Omega hosted by EMBL-EBI using the prepropeptide amino acid sequences of known nitrate- and rhizobia-responsive CLE peptides in M. truncatula, L. japonicus, soybean, common bean, pea and the root-specific CLE peptides of the non-legume Arabidopsis thaliana (Goujon et al., 2010; Sievers et al., 2011; McWilliam et al., 2013; Araya et al., 2014). In addition, new CLE 
BLASTN searches against the recently available pea genome (Kreplak et al., 2019). A phylogenetic tree was constructed from this alignment using the PhyML plugin in Geneious v.10.0.9, generating a tree based on the maximum likelihood method with 1,000 bootstraps supporting the branches and AtCLV3 as an outgroup (Guindon \& Gascuel, 2003). Microsynteny between genomic environments was assessed using Phytozome JBrowse. The orientation, predicted homologues and gene family of five genes directly up- and downstream were evaluated for each gene of interest, consistent with the methods used in Hastwell et al. (2015a).

\section{Sequencing}

Sanger sequencing (AGRF) was performed on M. truncatula A17 cDNA to confirm premature truncation of the MtCLE34 transcript. The CDNA was amplified using high-fidelity PrimeSTAR ${ }^{\circledR}$ Max DNA Polymerase (TaKaRa) followed by PCR clean-up. Primers were designed targeting the start codon and a region approximately $100 \mathrm{bp}$ past the predicted ATG-codon to account for signal loss ( $\pm 50 \mathrm{bp}$ ) during the sequencing process.

\section{Overexpression and Agrobacterium rhizogenes-mediated transformation}

The complete coding sequences of MtCLE12 (Medtr4g079630), MtCLE13 (Medtr4g079610) and MtCLE35 (Medtr2g091125) were cloned into the overexpression vector pCAMBIA1305.1. The PCR products were amplified from genomic DNA using PrimeSTAR ${ }^{\circledR}$ Max DNA Polymerase (TaKaRa). A restriction digest was performed using BgIII and BstEII (NEB) followed by ligation using the TaKaRa Mighty Mix ligation kit and heat-shock transformation of competent Escherichia coli XL1-Blue cells. Positive clones were confirmed using sequencing. The vectors, including the empty vector, were introduced into the A. rhizogenes ARqua1 strain using freeze-thaw transformation (Höfgen \& Willmitzer, 1988).

M. truncatula seedlings were transformed as described by Boisson-Dernier et al. (2001) and hairy roots were selected on modified Fåhraeus medium supplemented with the antibiotic hygromycin (3.5 $\mu \mathrm{g} / \mathrm{ml}$ ) for two weeks. After that, plantlets were transferred to nitrogen-free Fåhraeus medium and left for 5 days before inoculation with $10 \mu \mathrm{l}$ S. meliloti $1021(\mathrm{OD}=0.05)$. Nodules were counted at 10 dpi.

\section{Statistical analysis}


Statistical analyses were performed on all data using the Graphpad Prism v7 software. Following a Shapiro-Wilks normality test, a One-Way ANOVA was used to determine statistically significant differences in relative gene expression of the target genes compared with the respective controls. A Kruskal-Wallis test was used to determine significant differences in nodule number.

Results

MtCLE34 and MtCLE35 belong to distinct nodulation-suppressing clades

A phylogenetic tree using the pre-propeptide sequences of the nodulation-suppressing CLE peptides of M. truncatula, L. japonicus, soybean, common bean, pea and the non-legume $A$. thaliana, reveals three distinct nodulation-suppressing clades (Figure 1).

MTCLE34 is located in the green group (Figure 1) with the known nitrate-responsive CLE peptides, GmNIC1a/b and LjCLE40. The role of GmNIC1a/b in suppressing nodule organogenesis has been described previously, whereas there has not been conclusive overexpression evidence for the inhibitory role on nodulation of nitrate- and rhizobia-responsive LjCLE40 (Lim et al., 2011; Reid et al., 2011; Lim et al., 2014; Nishida et al., 2016). An uncharacterised pea orthologue, PsCam041632, also grouped in this clade indicating a potential role for it in nitrogen-response.

The orange group (Figure 1) contains MtCLE35 as well as rhizobia-responsive GmRIC1a/b, together with LjCLE-RS2 which is induced by both rhizobia and nitrate (Okamoto et al., 2009; Lim et al., 2011; Reid et al., 2011). GmRIC1a/b and LjCLE-RS2 are both widely known suppressors of legume nodulation. Interestingly, a pea orthologue is missing from this group. Since orthologues of genes in the green and orange clades are normally found in tandem pairs, we examined the genomic regions up- and downstream of PsCam041632 from the green group (Figure 2C). Microsynteny of surrounding genes was found to be completely lacking where the pea gene for this group would be expected. Similar investigations using the other nodulation-suppressing pea genes also failed to identify microsynteny. Hence, this region of genes, including the nodulation-suppressing CLE peptide encoding gene, was likely lost through a deletion event.

MtCLE13 is part of the blue group (Figure 1), which includes a member of every legume species investigated here (GmRIC2a/b, PvRIV2, LjCLE-RS1 and PsCAM040702). Within the blue group is a red subgroup (Figure 1) that includes MtCLE12, LjCLE5, the rhizobia- and nitrate-induced LjCLE-RS3, and 
two newly identified CLE peptides of pea, PsCam040984 and PsCam040153 (Mortier et al., 2010; Nishida et al., 2016; Hastwell et al., 2017). This subgroup does not contain any orthologues of soybean or common bean.

None of the reported nitrogen-regulated CLE peptides of $A$. thaliana were incorporated into the legume clades established here, which is consistent with previous reports (Figure 1; Hastwell et al., 2015a; 2017; 2019). Taken together, this may suggest that the nodulation-suppressing CLE peptides are unique to legumes, and that perhaps the nitrogen-regulated CLE peptides of $A$. thaliana are unique to this species, or to non-legumes in general.

The pre-propeptide sequences of CLE peptides are characterised by a signal peptide domain followed by a variable region and a mature CLE domain at the C-terminal that is highly conserved within gene families and species (Figure 2A; Hastwell et al., 2015b). However, MtCLE34 is predicted to be prematurely truncated. Sanger sequencing of MtCLE34 CDNA confirms the presence of a stop codon (TAG) in the variable region in front of the mature CLE domain. This would prevent the mature CLE peptide ligand from being translated and hence establishes that MtCLE34 is a pseudogene unable to produce a functional product.

Genomic microsynteny provides further evidence for a possible role of MtCLE34 and MtCLE35 in nodulation control (Figure 2B; 2C). The two genes are located in tandem on chromosome 2 in the $M$. truncatula genome, separated by only $9.6 \mathrm{~kb}$. This suggests a genetic duplication event occurred and that some degree of functional redundancy may exist with MtCLE35 covering for the lack of a functional copy of MtCLE34. This tandem duplication is true for all nodulation-suppressing CLE peptides of the model legumes $M$. truncatula, L. japonicus and soybean suggesting this early duplication occurred before species divergence followed by neofunctionalisation (Figure 2C; Hastwell et al., 2015a; 2017). Genomic regions, i.e., direction and function, are well conserved downstream of the MtCLE34/MtCLE35 gene pair when compared with PVNIC1/PVRIC1, GmNIC1a/GmRIC1b, GmNIC1b/GmRIC1a and LjCLE-RS2/LjCLE-RS3 (Figure 2B). MtCLE12 and MtCLE13 are found on chromosome 4 separated by $6.3 \mathrm{~kb}$ with a lower degree of microsynteny compared with other gene pairs.

MtCLE34 and MtCLE35 are induced by both nitrate and rhizobia

RT-qPCR was used to determine the expression patterns of MtCLE34 and MtCLE35 following nitrate treatment or rhizobia inoculation in wild-type roots. Both MtCLE34 and MtCLE35 expression were 
significantly upregulated by the addition of $2 \mathrm{mM}$ or $10 \mathrm{mM} \mathrm{KNO}_{3}$ relative to the no-nitrogen control $(P \leq 0.001$; Figure 3). Previously, concentrations of $2.5 \mathrm{mM}$ or greater were shown to significantly inhibit nodulation in $M$. truncatula, with the potassium in $\mathrm{KNO}_{3}$ having no effect on nodule numbers (van Noorden et al., 2016). The transcript abundance of both MtCLE34 and MtCLE35 gradually increased as the nitrate concentration increased (Figure 3). This is consistent with previous reports demonstrating a reverse correlation between increasing concentrations of available nitrate and reduced nodule numbers (Carroll et al., 1985; Barbulova et al., 2007; van Noorden et al., 2016). In contrast, expression of the known nodule-suppressing CLE peptide encoding genes, MtCLE12 and MtCLE13, was unaffected by nitrate treatment ( $P \geq 0.1$; Figure 3$)$.

In addition to nitrogen induction, the rhizobia-responsiveness of MtCLE12, MtCLE13, MtCLE34 and MtCLE35 was investigated by inoculating wild-type plants with S. meliloti, after which the zone of nodulation was harvested at $0,1,2,3,5$ and $7 \mathrm{dpi}$. As expected, MtCLE12 and MtCLE13 were induced by the bacteria, with a subtle difference in the timing of their onset (Figure 3; Mortier et al., 2010). MtCLE12 was induced at $5 \mathrm{dpi}$, reaching a peak in transcript abundance after which levels dropped off slightly ( $P \leq 0.001)$, while MtCLE13 was significantly upregulated as early as $2 \mathrm{dpi}$ with their transcript levels increasing up to $7 \mathrm{dpi}(P \leq 0.005$; Figure 3). The newly identified MtCLE35 was induced by rhizobia at $2 \mathrm{dpi}(P \leq 0.05)$, in a pattern similar to that of MtCLE13. Interestingly, despite MtCLE34 clustering with the CLE peptides acting in nitrate-dependent regulation of nodulation, it was also induced by rhizobia at 5 and $7 \mathrm{dpi}(P=0.02$ and $P \leq 0.001$ respectively; Figure 3$)$.

\section{MtCLE35 inhibits nodulation}

To determine whether MtCLE35 inhibits nodulation, overexpression of MtCLE35 using A. rhizogenesmediated hairy root transformation in a wild-type (A17) background was performed. MtCLE12 and MtCLE13 were also overexpressed. MtCLE34 overexpression was not assessed because it was confirmed here to be a pseudogene based on sequencing. Overexpression of all three CLE peptides reduced nodule numbers to a similar extent compared with the empty vector control $(P<0.001$; Figure 4) confirming a role for MtCLE35 in nodulation control.

\section{Discussion}

Nitrogen homeostasis is crucial for plant development and requires the integration of internal and external signals (e.g., nutrient status). Legumes tightly regulate their nodule number, size and nitrogen 
fixation rates in the presence of nitrogen (Streeter, 1988; Ferguson et al., 2019). While nitrogenresponsive CLE peptides have been previously identified in soybean, common bean and L. japonicus, they had not yet been characterised in M. truncatula, with only rhizobia-responsive CLE peptides reported (MtCLE12 and MtCLE13; Mortier et al., 2010). Moreover, pea orthologues of the nodulationsuppressing CLE peptides had not yet been thoroughly identified (Hastwell et al., 2019).

Our findings indicate that MtCLE12 and MtCLE13 have a distinct role in rhizobia-induced AON, whereas MtCLE35 can regulate nodule numbers in both a rhizobia- and nitrate-induced manner. Previous studies have shown that triarabinosylation of the peptides is required for SUNN-dependent nodule control (Schnabel et al., 2011; Kassaw et al., 2017; Imin et al., 2018). MtCLE34 likely had a similar role to MtCLE35, but the gene encoding it has been lost through a naturally-occurring mutation and a lack of evolutionary pressure that resulted in pseudogenisation and loss of the peptide ligand. Interestingly, L. japonicus also contains a mis-annotated pseudogene (LjCLE5) that is orthologous to MtCLE12 (Hastwell et al., 2017), although it is reported to not be expressed upon either rhizobia inoculation or nitrate treatment (Okamoto et al., 2009).

The nodulation-suppressing CLE peptides of legumes can be categorised into three distinct clades. The green clade contains MtCLE34, GmNIC1a/b, PVNIC1, PsCam041632 and LjCLE40. Indeed, all species explored here have a peptide in this group. They are induced by nitrate, with MtCLE34 and LjCLE4O also induced by rhizobia (Nishida et al., 2016).

The orange group contains MtCLE35, GmRIC1a/b, PvRIC1 and LjCLE-RS2. All of the genes encoding these peptides are induced by rhizobia, with MtCLE35 and LjCLE-RS2 also induced by nitrate (Okamoto et al., 2009; Reid et al., 2011; Nishida et al., 2016). Interestingly, pea seems to have lost its copy based on our bioinformatic analyses and genetic synteny investigations in the regions surrounding the other four CLE peptide genes of pea reported here.

The blue clade of nodulation-suppressing CLE peptides contains members that are only induced by rhizobia (i.e., MtCLE13, GmRIC2a/b, PvRIC2, and LjCLE-RS1, as well as PsCam040702). Through duplication events, this group led to the red subgroup that lack soybean and common bean orthologues (Okamoto et al., 2009; Mortier et al., 2010; Reid et al., 2011). The lack of these orthologues suggests that the red subgroup was either lost in soybean and bean, or originated in pea, M. truncatula and L. japonicus after the Phaseoloid clade diverged from the Hologaligena clade $>54$ million years ago (Lavin et al., 2005; Egan \& Doyle, 2010; Vanneste et al., 2014). 
323 Of the red subgroup, one branch contains MtCLE12, LjCLE5, and PsCam040153. The second contains LjCLE-RS3 and PsCam040984. Unlike the other members investigated to date, LjCLE-RS3 is the only one to also be induced by nitrate, but its induction is low compared to LjCLE-RS2 (Nishida et al., 2016). The missing $M$. truncatula CLE peptide in the second branch may have been lost, which is common for duplicated genes over time (Lynch \& Conery, 2000). Alternatively, it could be located in a part of the genome that has not been fully sequenced, but this seems unlikely as the $M$. truncatula genome has been thoroughly re-sequenced multiple times (Mt4.0v1; Tang et al., 2014).

Each of the legume species investigated here has CLE peptides in multiple clades. Their genes are often found directly adjacent on chromosomes, indicating they arose from tandem duplication(s) before speciation, after which neofunctionalisation occurred. These legume-specific peptides most likely evolved from existing CLE peptides acting in other developmental processes.

A role for small secreted peptides in macronutrient response was previously investigated by de Bang et al. (2017). In this study, MtCLE34 was downregulated in response to deficiencies in nitrogen, as well as phosphorus and sulphur, then was strongly upregulated following the re-supplementation of these macronutrients (de Bang et al., 2017). This indicates a more general role for MtCLE34 might have existed prior to it accumulating a null mutation. It is possible that MtCLE35 also responds to a number of macronutrients to regulate general root plasticity and nutrient homeostasis.

In A. thaliana, a number of CLE peptides have been identified that respond to nitrate. However, none of these CLE peptides group with the legume CLE peptides (Hastwell et al., 2017) and they conversely are upregulated following nitrogen deficiency (AtCLE1/3/4/7; Araya et al., 2014). Overexpression of these four peptides results in an inhibition of lateral root development (i.e., lateral root emergence), while the Atclv1 receptor mutant is characterised by increased lateral root length and density, indicating these peptides function in nitrogen foraging (Araya et al., 2014). Similarly, AON LRR-RLK mutants often have aberrant root phenotypes that include shorter primary roots and higher lateral root density (e.g., Wopereis et al., 2000; Schnabel et al., 2005). In addition, some root architecture responses to nitrate are differentially affected in the M. truncatula AON mutants (Goh et al., 2018). upregulated by rhizobia but not by nitrate treatment. MtCLE13 groups closely with the rhizobiaresponsive CLE peptides GmRIC2a/b and LjCLE-RS1. MtCLE12 forms a clade with LjCLE-RS3, which 
seems to act differently than the other nodulation-suppressing CLE peptides. Indeed, both MtCLE12 and LjCLE-RS3 are upregulated at a later stage of nodulation with the latter also being induced in the presence of nitrogen and rhizobia. LjCLE-RS3 overexpression also results in a weaker inhibition of nodulation compared with LjCLE-RS1 and LjCLE-RS2 (Nishida et al., 2016). While MtCLE13 is nodulespecific, MtCLE12 expression also occurs in the root tip, cotyledons and first leaves (Mortier et al., 2010). In addition, it is not induced by exogenous application of cytokinin, unlike MtCLE13 (Mortier et al., 2010). Both cytokinin and the consequent upregulation of NODULE INCEPTION (NIN), are crucial for nodule primordia establishment and infection (Murray et al., 2007; Tirichine et al., 2007; Plet et al., 2011). NIN has also been shown to bind to the promoter region of these CLE peptides linking it to AON and nitrate-dependent control of nodulation (Soyano et al., 2014; 2015; Lin et al., 2018; Nishida et al., 2018).

Maintaining multiple CLE peptides able to regulate nodule numbers must be important for effective control of nodulation and hence why legume species have retained so many throughout evolution. Subtle temporal differences in the expression of the nodulation-suppressing CLE peptides indicates that timing of expression is also crucial. More work is now needed to understand the subtle and sophisticated roles each of these peptide hormones play in nodulation control, and possibly also in other aspects of plant development and nutrient response.

\section{Author contributions}

$\mathrm{BF}$ devised the project and $\mathrm{BF}$ and $\mathrm{PM}$ supervised. $\mathrm{CM}$ and $\mathrm{AH}$ performed bioinformatic analyses. $\mathrm{HS}$ assisted with cloning and UM conducted the overexpression experiment. CM performed gene expression analysis, analysed the data, prepared figures and wrote the manuscript with BF and additional input from all authors. All authors read and approved the manuscript.

\section{Acknowledgements}

This work was funded by the Australian Research Council (ARC) Grants DP130102266, DP130103084 and DP190102996; and the Hermon Slade Foundation. CM is supported by an RTP Scholarship. AHH

382 is funded by an ARC Discovery Early Career Research Award (DE200100800) and the Molly-Budtz Olsen

383 Fellowship from The Fellowship Fund Inc. We would like to acknowledge Bowen Harding and Jason Ng for their technical assistance. 


\section{References}

Araya T, Miyamoto M, Wibowo J, Suzuki A, Kojima S, Tsuchiya YN, Sawa S, Von Wirén N, Takahashi H. 2014. CLE-CLAVATA1 peptide-receptor signaling module regulates the expansion of plant root systems in a nitrogen-dependent manner. Proceedings of the National Academy of Sciences 111(5): 2029-2034.

Barbulova A, Rogato A, d'Apuzzo E, Omrane S, Chiurazzi M. 2007. Differential effects of combined sources on early steps of the nod factor-dependent transduction pathway in Lotus japonicus. Molecular Plant-Microbe Interactions 20: 994-1003.

Boisson-Dernier A, Chabaud M, Garcia F, Bécard G, Rosenberg C, Barker DG. 2001. Agrobacterium rhizogenes-transformed roots of Medicago truncatula for the study of nitrogen-fixing and endomycorrhizal symbiotic associations. Molecular Plant-Microbe Interactions Journal 14(6): 695-700.

Caetano-Anollés G, Gresshoff PM. 1991. Alfalfa controls nodulation during the onset of Rhizobiuminduced cortical cell division. Plant Physiology 95: 366-373.

Caetano-Anollés G, Gresshoff PM. 1991. Plant genetic control of nodulation. Annual Review of Microbiology 45: 345-382.

Carroll BJ, McNeil DL, Gresshoff PM. 1985. A supernodulation and nitrate-tolerant symbiotic (nts) soybean mutant. Plant Physiology 78: 34-40.

Corcilius L, Hastwell AH, Zhang M, Williams J, Mackay JP, Gresshoff PM, Ferguson BJ, Payne RJ. 2017. Arabinosylation modulates the growth-regulating activity of the peptide hormone CLE40a from soybean. Cell Chemical Biology 24: 1-9.

de Bang TC, Lundquist PK, Dai X, Boschiero C, Zhuang Z, Pant P, Torres-Jerez I, Roy S, Nogales J, Veerappan V, et al. 2017. Genome-wide identification of Medicago peptides involved in macronutrient responses and nodulation. Plant Physiology 175(4): 1669-1689.

Delves AC, Mathews A, Day DA, Carter AS, Carroll BJ, Gresshoff PM. 1986. Regulation of the soybeanRhizobium nodule symbiosis by shoot and root factors. Plant Physiology 82: 588-590.

Egan AN, Doyle J. 2010. A comparison of global, gene-specific, and relaxed clock methods in a comparative genomics framework: dating the polyploid history of soybean (Glycine max). Systematic Biology 59(5): 534-547.

Fåhraeus G. 1957. The infection of clover root hairs by nodule bacteria studied by a simple glass slide technique. Journal of General Microbiology 16: 374-381.

Ferguson BJ, Li D, Hastwell AH, Reid DE, Li Y, Jackson SA, Gresshoff PM. 2014. The soybean (Glycine max) nodulation-suppressive CLE peptide, GmRIC1, functions interspecifically in common 
white bean (Phaseolus vulgaris), but not in a supernodulating line mutated in the receptor PVNARK. Plant Biotechnol J 12(8): 1085-1097.

Ferguson BJ, Mens C, Hastwell AH, Zhang M, Su H, Jones CH, Chu X, Gresshoff PM. 2019. Legume nodulation: The host controls the party. Plant, Cell \& Environment 42(1): 41-51.

Fletcher JC, Brand U, Running MP, Simon R, Meyerowitz EM. 1999. Signaling of cell fate decisions by CLAVATA3 in Arabidopsis shoot meristems. Science 283(5409): 1911-1914.

Funayama-Noguchi S, Noguchi K, Yoshida C, Kawaguchi M. 2011. Two CLE genes are induced by phosphate in roots of Lotus japonicus. Journal of Plant Research 124(1): 155-163.

Gautrat P, Laffont C, Frugier F. 2020. Compact Root Architecture 2 promotes root competence for nodulation through the miR2111 systemic effector. Current Biology 30(7): 1339-1345.

Goh C-H, Nicotra AB, Mathesius U. 2018. Genes controlling legume nodule numbers affect phenotypic plasticity responses to nitrogen in the presence and absence of rhizobia. Plant, Cell and Environment 42(5): 1747-1757.

Goujon M, McWilliam H, Li W, Valentin F, Squizzato S, Paern J, Lopez R. 2010. A new bioinformatics analysis tools framework at EMBL-EBI. Nucleic Acids Research 38: W695-W699.

Gresshoff PM, Skotnicki ML, Eadie JF, Rolfe BG. 1977. Viability of Rhizobium trifolii bacteroids from clover root nodules. Plant Science Letters 10: 209-304.

Guindon S, Gascuel O. 2003. A simple, fast, and accurate algorithm to estimate large phylogenies by maximum likelihood. Systematic Biology 52(5): 696-704.

Hastwell AH, Corcilius L, Williams J, Gresshoff PM, Payne RJ, Ferguson BJ. 2019. Triarabinosylation is required for nodulation-suppressive CLE peptides to systemically inhibit nodulation in Pisum sativum. Plant, Cell \& Environment 42(1): 188-197.

Hastwell AH, de Bang TC, Gresshoff PM, Ferguson BJ. 2017. CLE peptide-encoding gene families in Medicago truncatula and Lotus japonicus, compared with those of soybean, common bean and Arabidopsis. Scientific Reports 7(1): 9384.

Hastwell AH, Gresshoff PM, Ferguson BJ. 2015a. Genome-wide annotation and characterization of CLAVATA/ESR (CLE) peptide hormones of soybean (Glycine max) and common bean (Phaseolus vulgaris), and their orthologues of Arabidopsis thaliana. Journal of Experimental Botany 66(17): 5271-5287.

Hastwell AH, Gresshoff PM, Ferguson BJ. 2015b. The structure and activity of nodulation-suppressing CLE peptide hormones of legumes. Functional Plant Biology 42(3): 229-238.

Hobe M, Müller R, Grünewald M, Brand U, Simon R. 2003. Loss of CLE40, a protein functionally equivalent to the stem cell restricting signal CLV3, enhances root waving in Arabidopsis. Development Genes and Evolution 213(8): 371-381. 
Höfgen R, Willmitzer L. 1988. Storage of competent cells for Agrobacterium transformation. Nucleic Acids Res 16(20): 9877.

Imin N, Patel N, Corcilius L, Payne RJ, Djordjevic MA. 2018. CLE peptide tri-arabinosylation and peptide domain sequence composition are essential for SUNN-dependent autoregulation of nodulation in Medicago truncatula. New Phytol 218(1): 73-80.

Ito Y, Nakanomyo I, Motose H, Iwamoto K, Sawa S, Dohmae N, Fukuda H. 2006. Dodeca-CLE peptides as suppressors of plant stem cell differentiation. Science 313(5788): 842-845.

Karlo M, Boschiero C, Landerslev KG, Blanco GS, Wen J, Mysore KS, Dai X, Zhao PX, de Bang TC. 2020. The CLE53-SUNN genetic pathway negatively regulates arbuscular mycorrhiza root colonization in Medicago truncatula. Journal of Experimental Botany. eraa193

Kassaw T, Nowak S, Schnabel E, Frugoli J. 2017. ROOT DETERMINED NODULATION1 is required for $M$. truncatula CLE12, but not CLE13, peptide signaling through the SUNN receptor kinase. Plant Physiology 174(4): 2445-2456.

Kreplak J, Madoui MA, Cápal P, Novák P, Labadie K, Aubert G, Bayer PE, Gali KK, Syme RA, Main D, et al. 2019. A reference genome for pea provides insight into legume genome evolution. Nature Genetics 51(9): 1411-1422.

Krusell L, Madsen LH, Sato S, Aubert G, Genua A, Szczyglowski K, Duc G, Kaneko T, Tabata S, de Bruijn F, et al. 2002. Shoot control of root development and nodulation is mediated by a receptorlike kinase. Nature 420: 422-426.

Lavin M, Herendeen PS, Wojciechowski MF. 2005. Evolutionary rates analysis of Leguminosae implicates a rapid diversification of lineages during the tertiary. Systematic Biology 54(4): 575594.

Lim CW, Lee YW, Hwang CH. 2011. Soybean nodule-enhanced CLE peptides in roots act as signals in GmNARK-mediated nodulation suppression. Plant Cell Physiol 52(9): 1613-1627.

Lin J-S, Li X, Luo ZL, Mysore KS, Wen J, Xie F. 2018. NIN interacts with NLPS to mediate nitrate inhibition of nodulation in Medicago truncatula. Nature Plants 4: 942-952.

Lynch M, Conery JS. 2000. The evolutionary fate and consequences of duplicate genes. Science $\mathbf{2 9 0 :}$ 1151-1155.

McWilliam H, Li W, Uludag M, Squizzato S, Park YM, Buso N, Cowly AP, Lopez R. 2013. Analysis tool web services from the EMBL-EBI. Nucleic Acids Research 41: W597-W600.

Miller AJ, Fan X, Orsel M, Smith SJ, Wells DM. 2007. Nitrate transport and signalling. Journal of Experimental Botany 58(9): 2297-2306. 
Mortier V, Den Herder G, Whitford R, Van de Velde W, Rombauts S, D'haeseleer K, Holsters M, Goormachtig S. 2010. CLE peptides control Medicago truncatula nodulation locally and systemically. Plant Physiology 153(1): 222-237.

Murray JD, Karas BJ, Sato S, Tabata S, Amyot L, Szczyglowski K. 2007. A cytokinin perception mutant colonized by Rhizobium in the absence of nodule organogenesis. Science 315(5808): 101-104.

Nishida H, Handa Y, Tanaka S, Suzaki T, Kawaguchi M. 2016. Expression of the CLE-RS3 gene suppresses root nodulation in Lotus japonicus. Journal of Plant Research 129(5): 909-919.

Nishida H, Ito M, Miura K, Kawaguchi M, Suzaki T. 2020. Autoregulation of nodulation pathway is dispensable for nitrate-induced control of rhizobial infection. Plant Signaling \& Behavior 15(3): 1733814.

Nishida H, Tanaka S, Handa Y, Ito M, Sakamoto Y, Matsunaga S, Betsuyaky S, Miura K, Soyano T, Kawaguchi M, et al. 2018. A NIN-LIKE PROTEIN mediates nitrate-induced control of root nodule symbiosis in Lotus japonicus. Nature Communications 9(1): 499.

Nishimura R, Hayashi M, Wu GJ, Kouchi H, Imaizumi-Anraku H, Murakami Y, Kawasaki S, Akao S, Ohmori M, Nagasawa M, et al. 2002. HAR1 mediates systemic regulation of symbiotic organ development. Nature 420(6914): 426-429.

Okamoto S, Ohnishi E, Sato S, Takahashi H, Nakazono M, Tabata S, Kawaguchi M. 2009. Nod factor/nitrate-induced CLE genes that drive HAR1-mediated systemic regulation of nodulation. Plant Cell Physiology 50(1): 67-77.

Okamoto S, Shinohara H, Mori T, Matsubayashi Y, Kawaguchi M. 2013. Root-derived CLE glycopeptides control nodulation by direct binding to HAR1 receptor kinase. Nature Communications 4: 2191.

Plet J, Wasson A, Ariel F, Le Signor C, Baker D, Mathesius U, Crespi M, Frugier F. 2011. MtCRE1dependent cytokinin signaling integrates bacterial and plant cues to coordinate symbiotic nodule organogenesis in Medicago truncatula. The Plant Journal 65(4): 622-633.

Reid DE, Ferguson BJ, Gresshoff PM. 2011. Inoculation- and nitrate-induced CLE peptides of soybean control NARK-dependent nodule formation. Molecular Plant-Microbe Interactions 24(5): 606618.

Schnabel E, Journet EP, de Carvalho-Niebel F, Duc G, Frugoli J. 2005. The Medicago truncatula SUNN gene encodes a CLV1-like leucine-rich repeat receptor kinase that regulates nodule number and root length. Plant Molecular Biology 58(6): 809-822.

Schnabel EL, Kassaw TK, Smith LS, Marsh JF, Oldroyd GE, Long SR, Frugoli JA. 2011. The ROOT DETERMINED NODULATION1 gene regulates nodule number in roots of Medicago truncatula 
and defines a highly conserved, uncharacterized plant gene family. Plant Physiology 157(1): 328-340.

Searle IR, Men AE, Laniya TS, Buzas DM, Iturbe-Ormaetxe I, Carol BJ, Gresshoff PM. 2003. Longdistance signalling in nodulation directed by a CLAVATA1-like receptor kinase. Science 299(5603): 109-112.

Shinohara H, Matsubayashi Y. 2013. Chemical synthesis of Arabidopsis CLV3 glycopeptide reveals the impact of hydroxyproline arabinosylation on peptide conformation and activity. Plant and Cell Physiology 54(3): 369-374.

Sievers F, Wilm A, Dineen D, Gibson TJ, Karplus K, Li W, Lopez R, McWilliam H, Remmert M, Söding J, et al. 2011. Fast, scalable generation of high-quality protein multiple sequence alignments using Clustal Omega. Molecular Systems Biology 7(1): 539.

Soyano T, Hirakawa H, Sato S, Hayashi M, Kawaguchi M. 2014. NODULE INCEPTION creates a longdistance negative feedback loop involved in homeostatic regulation of nodule organ production. Proceedings of the National Academy of Sciences of the United States of America 111(40): 14607-14612.

Soyano T, Shimoda Y, Hayashi M. 2015. NODULE INCEPTION antagonistically regulates gene expression with nitrate in Lotus japonicus. Plant Cell Physiology 56(2): 368-376.

Streeter J. 1988. Inhibition of legume nodule formation and $\mathrm{N}_{2}$ fixation by nitrate. Critical Reviews in Plant Sciences 7(1): 1-23.

Takahara M, Magori S, Soyano T, Okamoto S, Yoshida C, Yano K, Sato S, Tabata S, Yamaguchi K, Shigenobu S, et al. 2013. TOO MUCH LOVE, a novel Kelch repeat-containing F-box protein, functions in the long-distance regulation of the legume-Rhizobium symbiosis. Plant and Cell Physiology 54(4): 433-447.

Tang H, Krishnakumar V, Bidwell S, Rosen B, Chan A, Zhou S, Gentzbittel L, Childs KL, Yandell M, Gundlach $\mathrm{H}$, et al. 2014. An improved genome release (version Mt4.0) for the model legume Medicago truncatula. BMC Genomics 15: 312.

Tirichine L, Sandal N, Madsen LH, Radutoiu S, Albrektsen AS, Sato S, Asamizu E, Tabata S, Stougaard J. 2007. A gain-of-function mutation in a cytokinin receptor triggers spontaneous root nodule organogenesis. Science 315(5808): 104-107.

Tsikou D, Yan Z, Holt DB, Abel NB, Reid DE, Madsen LH, Bhasin H, Sexauer M, Stougaard J, Markmann K. 2018. Systemic control of legume susceptibility to rhizobial infection by a mobile microRNA. Science 362(6411): 233-236. 
van Noorden GE, Verbeek R, Dinh QD, Jin J, Green A, Ng JLP, Mathesius U. 2016. Molecular signals controlling the inhibition of nodulation by nitrate in Medicago truncatula. International Journal of Molecular Sciences 17(7): 1060.

552

Vanneste K, Baele G, Maere S, Van de Peer Y. 2014. Analysis of 41 plant genomes supports a wave of successful genome duplications in association with the Cretaceous-Paleogene boundary. Genome Research 24(8): 1334-1347. 


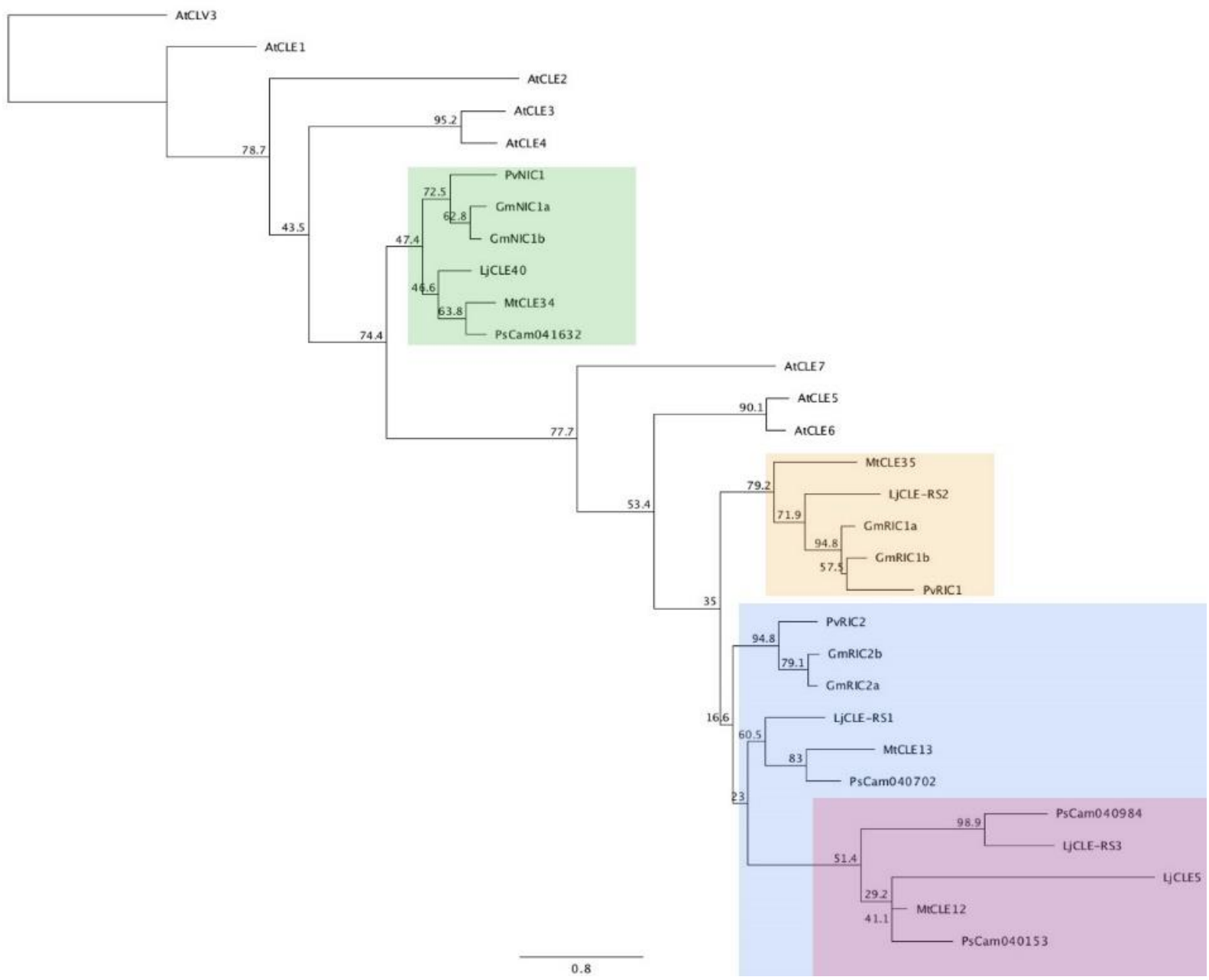

Figure 1. Phylogenetic tree of nitrogen- and/or rhizobia-induced CLE peptides of Medicago truncatula, Lotus japonicus, soybean, common bean, pea and Arabidopsis thaliana. The full prepropeptide sequence of each was used. AtCLV3 was added as an outgroup. Three distinct clades (green, orange and blue), with a subgroup (red) were established for the legume sequences. Bootstrap confidence values are shown as percentages from 1,000 bootstrap replications. 


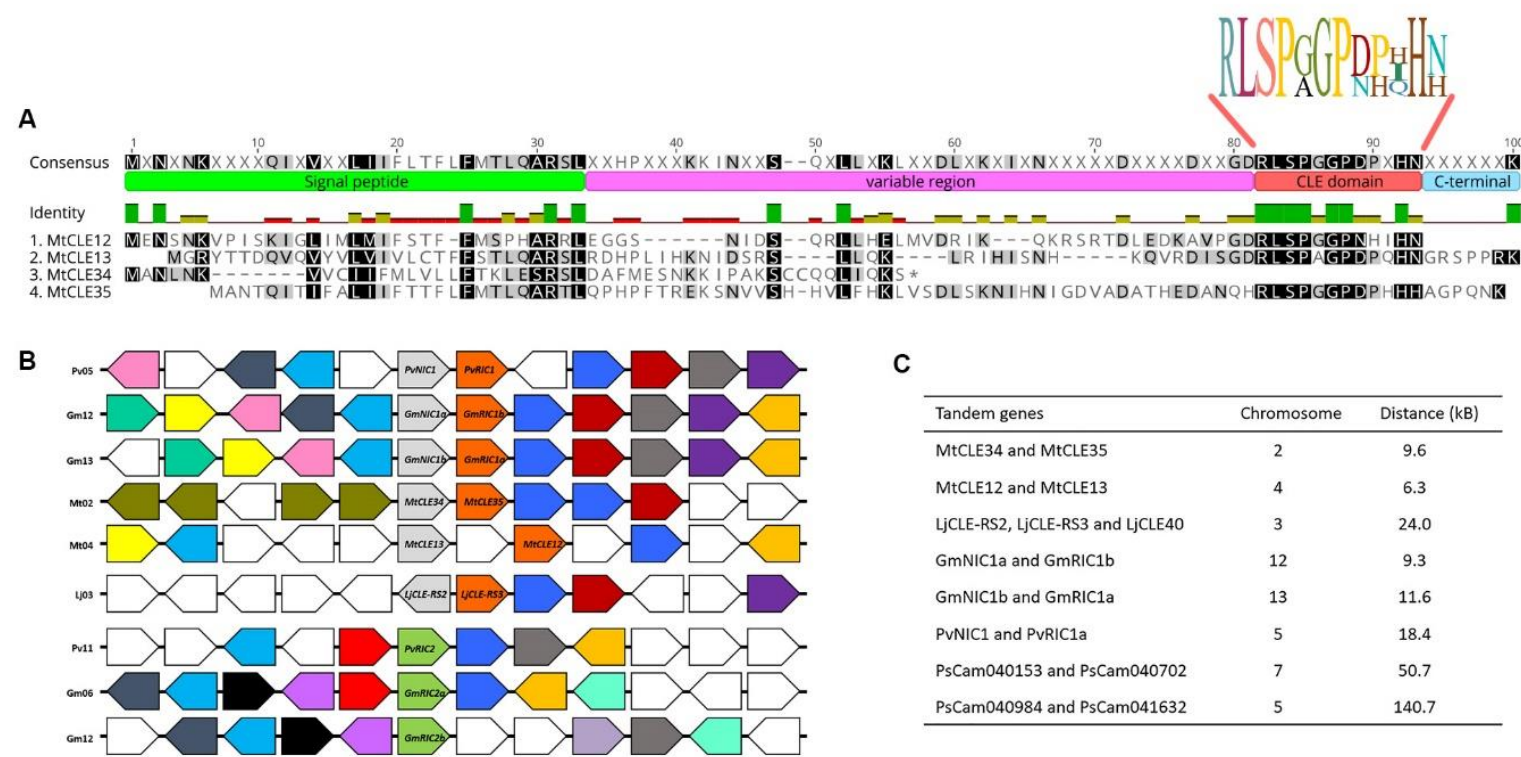

Figure 2. Bioinformatic analysis of nodulation-suppressing CLE orthologues in Medicago truncatula, among residues with the darker the shading, the higher the level of residue conservation. A sequence logo generated in Geneious v.10.0.9 is shown for the mature CLE peptide. $\left({ }^{*}\right)$ represents a predicted truncation before the mature MtCLE34 peptide. The multiple sequence alignment was generated using Clustal Omega hosted by EMBL-EBI and visualized using Geneious v.10.0.9. (B) Microsynteny of nodulation-suppressive CLE peptides in soybean, common bean, M. truncatula and L. japonicus. The genes of interest are positioned centrally with arrows indicating the direction of surrounding genes relative to the genes of interest. Genes with a similar putative gene function are shown in the same colours and those with an unrelated or unknown function left uncoloured. (C) Chromosomal location and distance between CLE gene pairs in M. truncatula, L. japonicus, soybean, common bean and pea. 
A
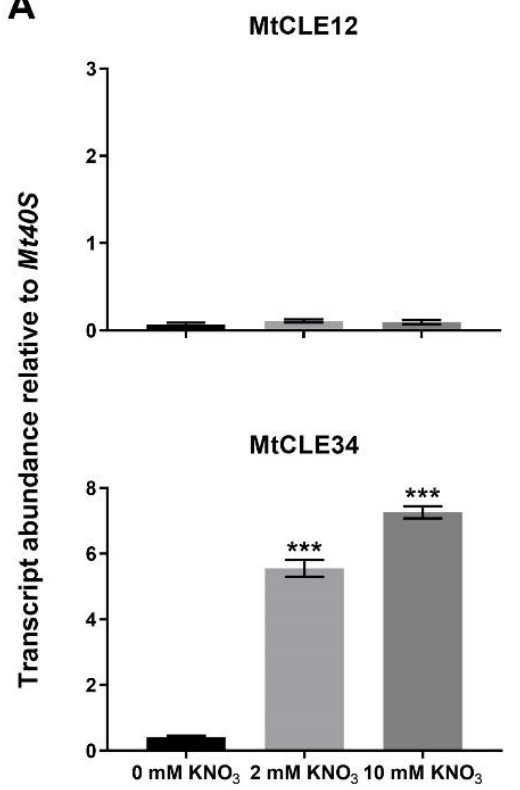

B

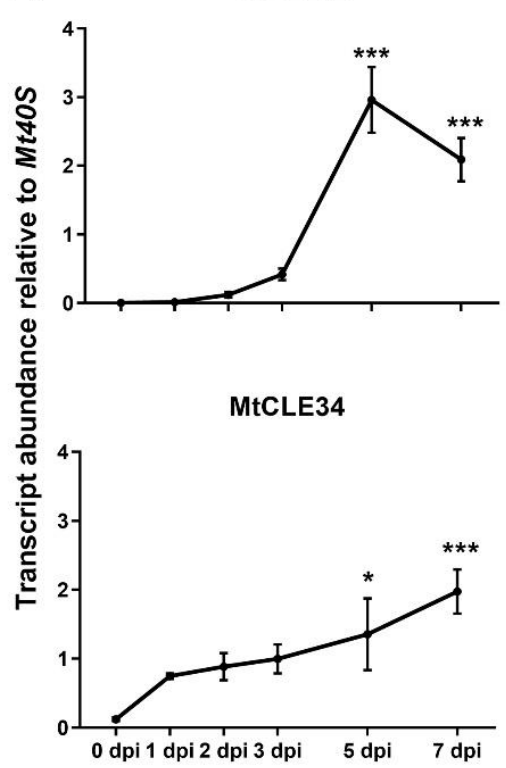

MtCLE13

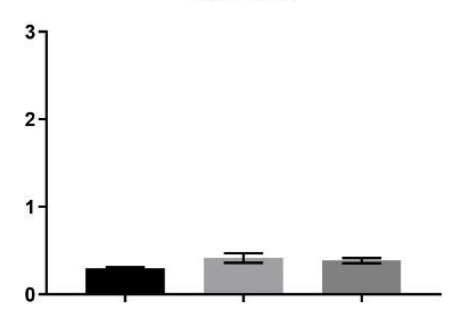

MtCLE35

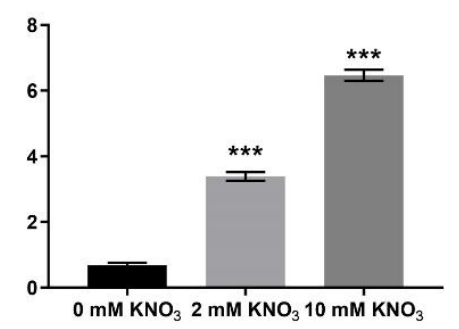

MtCLE13
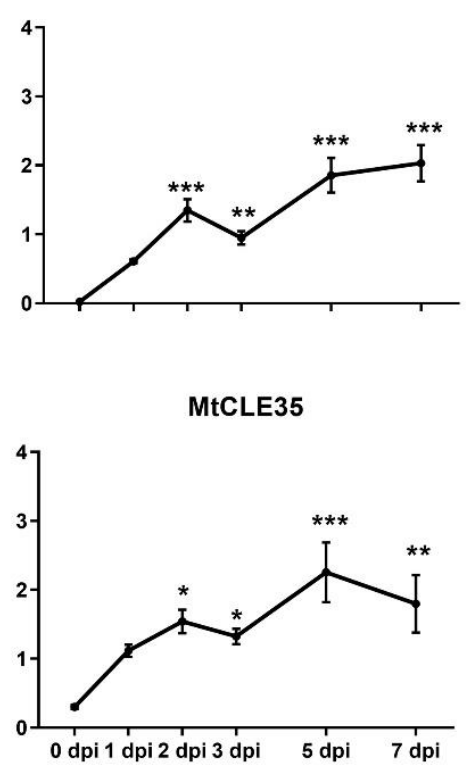
were harvested. (B) Following inoculation with Sinorhizobium meliloti, the zone of nodulation above the root tip was harvested at $0,1,2,3,5$ and 7 days post inoculation (dpi). Transcript abundance is relative to the housekeeping gene Mt40s ribosomal protein S8. Bars represent means \pm SEM of at least two biological replicates ( $n=11-14$ per replicate). Asterisks indicate significant differences in transcript abundance compared to the control (One-Way ANOVA, ${ }^{* * *} P \leq 0.001, * * P \leq 0.01,{ }^{*} P \leq 0.05$ ). 


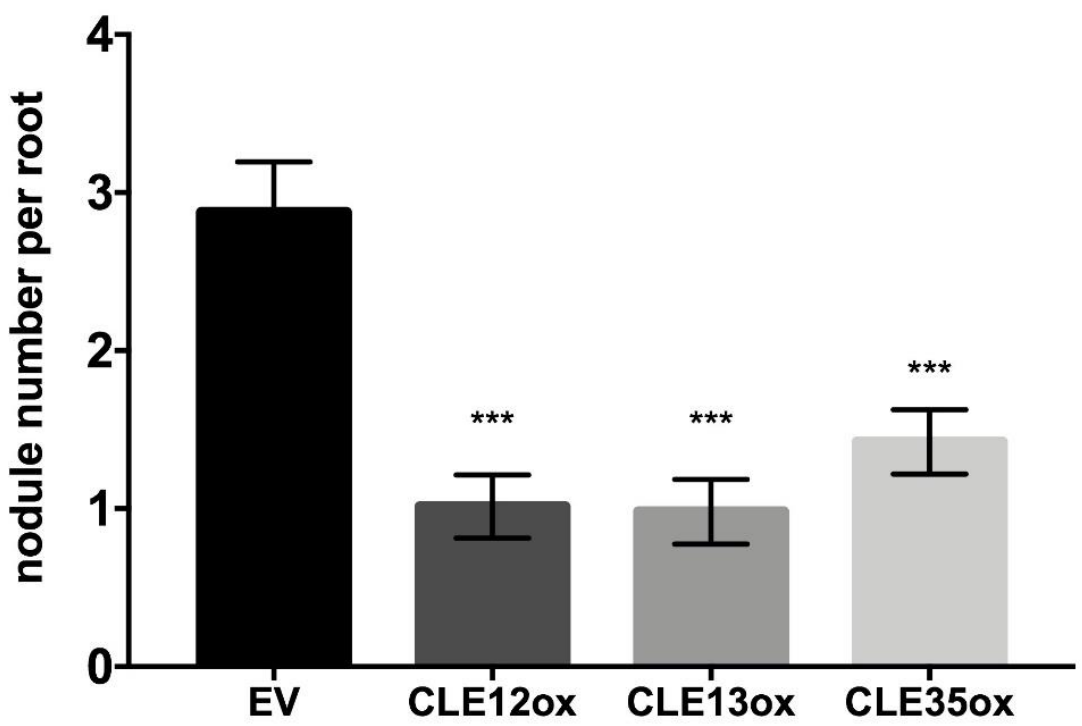

599 Figure 4. Nodule numbers of wild-type A17 plants overexpressing MtCLE12, MtCLE13 or MtCLE35.

600 Bars represent means \pm SEM. Asterisks indicate significant differences in nodule number compared to 601 the empty vector control $\left(n=48-78 ;\right.$ Kruskal-Wallis, $\left.{ }^{* * *} P \leq 0.001\right)$.

602

603 